\title{
An Unusual Case of Stroke: A Case Report and Review of Current Literature
}

\author{
Alistair Mackett, Saugata Das, Muhibbur Chowdhury, Peter Phillips \\ Ipswich Hospital, Ipswich, UK \\ Email: alistair.mackett@ipswichhospital.nhs.uk
}

Received 9 April 2015; accepted 30 May 2015; published 3 June 2015

Copyright (C) 2015 by authors and Scientific Research Publishing Inc.

This work is licensed under the Creative Commons Attribution International License (CC BY). http://creativecommons.org/licenses/by/4.0/

(c) (†) Open Access

\begin{abstract}
Fibromuscular dysplasia (FMD) is a non-atherosclerotic, non-inflammatory vascular disease. FMD once considered rare, is increasingly a condition recognised as an incidental finding. It is an important cause of stroke especially in younger, female patients and therefore a high index of suspicion is required. FMD has distinct pathological findings along with some pathognomonic angiographic findings ("string of beads" appearance). The mainstay of cerebrovascular FMD treatment is antiplatelet therapy but intervention with percutaneous transluminal angioplasty is indicated under some circumstances. We present an interesting case of a woman in her $60 \mathrm{~s}$ who underwent extensive investigation to identify the underlying aetiology of her stroke.
\end{abstract}

\section{Keywords}

Fibromuscular Displasia, Stroke

\section{Introduction}

Fibromuscular dysplasia (FMD) is a non-atherosclerotic, non-inflammatory vascular disease [1], which has been observed in almost every artery throughout the body, however it is more typically associated with renal and carotid arteries [2]. The typical renal FMD presentation is hypertension whereas carotid/vertebral FMD has been associated with stroke and TIA. We present a case to illustrate how FMD, although not being an uncommon cause of stroke, is significantly under-recognised as a high index of suspicion required to diagnose it. The subsequent discussion will focus on the aetiology, diagnosis and management of carotid and vertebral FMD.

\section{Case Presentation}

A female office worker in her early $60 \mathrm{~s}$ attended our emergency department (ED) having developed left facial

How to cite this paper: Mackett, A., Das, S., Chowdhury, M. and Phillips, P. (2015) An Unusual Case of Stroke: A Case Report and Review of Current Literature. Open Journal of Internal Medicine, 5, 5-10. 
asymmetry, left sided weakness, and left visual neglect. Our hospital is representative of a medium to large district general hospital in England treating about 600 inpatient strokes per year. The onset time was not clear as she had assumed it would resolve and continued working until her family encouraged her to seek medical attention. On review it became apparent she had a paucity of risk factors for cerebrovascular disease. She had no relevant past medical or family history, was a life-long non-smoker, drank alcohol very occasionally and was generally very active with a normal body mass index (BMI). Examination revealed profound left visual neglect, left upper motor neurone facial palsy and dysarthric speech. She had a mild left hemiparaesis with grade 4/5 power on the left. Her cardiovascular examination was normal.

The working diagnosis was a right partial anterior circulation stroke. She had an initial CT (see Figure 1), which demonstrated an area of low attenuation in the right parietal lobe consistent with an infarct after her delayed presentation. She went on to have a MRI brain with diffusion weighted sequence (see Figure 2) to confirm an is chaemic stroke and exclude other pathology such as a tumour. She underwent extensive investigations to identify the underlying cause of her stroke. Her ECG and ambulatory monitor showed no evidence of atrial fibrillation and she had a normal echocardiogram. There was no evidence of hypertension, hyperlipidaemia or diabetes mellitus. Rarer causes such as lupus or thrombophilia were also excluded. No evidence of significant internal carotid artery stenosis was identified on carotid doppler ultrasound scanning.

A further magnetic-resonance angiogram (MRA) of her carotids was organised as a second imaging modality (see Figure 3). This demonstrated a "string of beads" appearance and she was diagnosed with fibromuscular dysplasia. (FMD). The patient was subsequently referred to a tertiary centre for a conventional angiogram of the cerebral vessels. After discussion in the neurosurgical centre's multi-disciplinary team meeting she was not deemed appropriate for surgical or radiological intervention at present. Instead she was commenced on dual antiplatelet therapy (aspirin and clopidogrel). The patient made a good functional recovery and was left with only a mild visual field defect that was noted during her follow up appointments. Formal written consent for this case report was obtained from the patient.

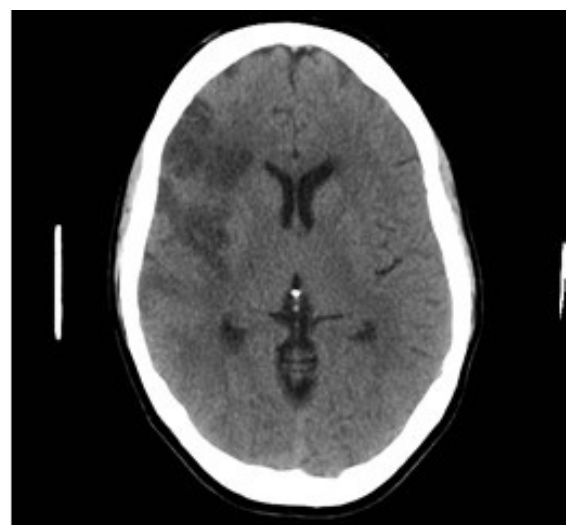

Figure 1. CT head FMD.

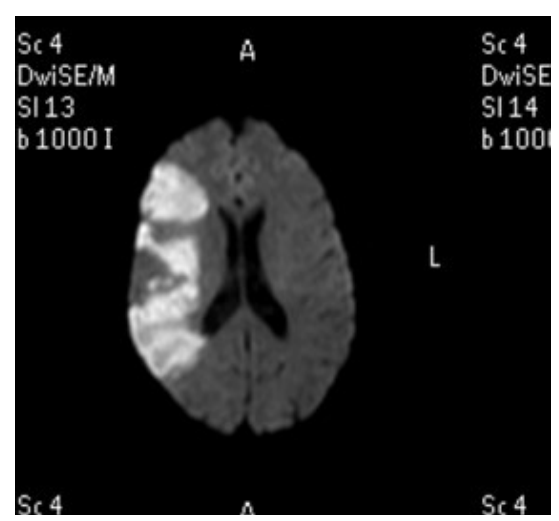

Figure 2. MRI FMD. 


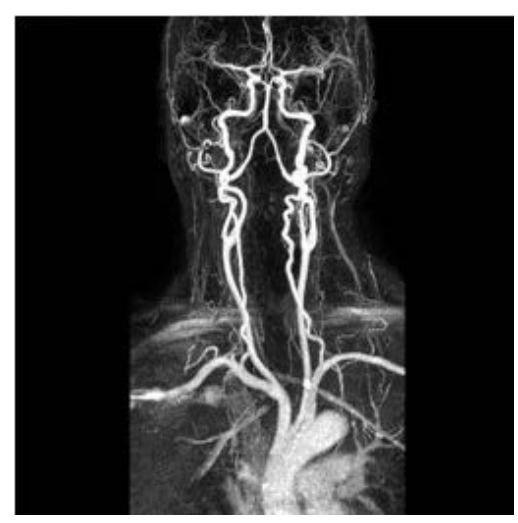

Figure 3. MRA FMD.

\section{Discussion}

\subsection{Epidemiology and Aetiology}

Once thought vanishingly rare FMD is now an increasingly incidental finding. Several studies looking at potential renal donors have found incidental rates between $2 \%-6.6 \%$ on renal angiogram [3]-[5]. Up to $90 \%$ of those affected are female [6]. The exact underlying aetiology of FMD is currently unknown. There may be an association with smoking [7] however the general consensus is there is likely to be an underlying genetic component. In the US registry for fibromuscular dysplasia 7.3\% had a confirmed diagnosis of FMD among a family member and $23.5 \%$ had a family history of aneurysm [6]. Due to the rarity of FMD there are no large cohorts for genetic studies and small case control studies are yet to identify a consistent genetic mutation.

Leadbetter and Burkland first mentioned the pathology of FMD in the literature in 1938 when they described curing a 5-year-old boy of hypertension following a unilateral nephrectomy [8]. It was not until 1958 when McCormack et al gave the first accurate pathological description of FMD with a series of four patients with reno-vascular hypertension [9].

\subsection{Pathology}

There are three main sub-types of FMD: intima, media and adventitia [10] [11].

\subsubsection{Medial}

This is the most common histological variant representing over $90 \%$ of identified cases. Fibromuscular ridges are formed which lead to arterial stenoses alternating with area of smooth muscle loss with consequent arterial dilation giving the typical "string of beads" pattern seen on angiography.

\subsubsection{Intimal}

This is the second most commonly encountered type of FMD as represented by focal angiographic stenoses. Essentially there is accumulation of fibrous tissue in the intima.

\subsubsection{Adventitial}

This represents fewer than 1\% of FMD cases and consists of collagen deposition surrounding the adventitia and extending into the periarterial tissue. Once again this leads to focal stenoses seen on angiography.

As histopathology specimens for diagnosis of FMD become less frequent there has been a move to define FMD by its angiographic appearances. The French and Belgian consensus statement in 2012 defined three angiographic appearances: multifocal, unifocal and tubular [2] and the American Heart Association in 2014 further refined the classification into multifocal and focal [12]. Multifocal lesions correspond to medial histopathology whereas focal lesions corresponded to intimal or adventitial histopathology.

\subsection{Clinical Presentation of Cerebrovascular FMD}

The presentation of cerebrovascular FMD is very variable and non-specific therefore it can be a challenging di- 
agnosis to reach. There are a proportion of patients with asymptomatic FMD [13]. The US registry identified the commonest symptoms of FMD, which include hypertension (63.8\%) headache (52.4\%) followed by pulsatile tinnitus (27.5\%). The frequency of neurological sequelea in FMD is significant with rates of stroke and TIA $9.8 \%$ and $13.4 \%$ respectively. $12.1 \%$ had suffered a cervical artery dissection and there was an increase in the incidence of subarachnoid haemorrhage [6]. FMD has a number of manifestations including: severe stenosis (haemodynamic strokes), thromobosis, emboli, dissection and aneurysm rupture leading to focal neurological events [12]. Prevalence of intracranial aneuryms may be as high as $21 \%$ [13], but a more recent paper revises the estimate down to $7.3 \%$ [14].

\subsection{Diagnosis}

Many patients who present with stroke will undergo carotid artery duplex scanning however the identification of FMD can be difficult and is user dependent. In renal artery stenosis ultrasound only has a $60 \%$ positive predictive value [15]. Typical findings include beading and tortuosity of the mid and distal internal carotid artery. Often the main clue is the site of the stenosis as atheromatous disease tends to be seen more proximally therefore a high index of suspicion is required to correctly identify cases. Both CT angiography (CTA) and MRA are widely used in the diagnosis of FMD. CTA and MRA have been validated against catheter-based angiography in the context of atheromatous disease with a correlation rate of in excess of 97\% CTA and 95\% MRA however there are no studies defining either accuracy in FMD [16]. Although MRA has similar correlation rates to CTA it does have some advantages including being more likely to identify FMD related dissection [17]. The changes seen in the arterial wall result in a number of common angiographic appearances: focal stenosis, tubular stenosis, multifocal stenosis alternating with mural dilatation ("string of beads" appearance), a septum or diverticulum. Catheter based angiography remains the gold standard however its use is now reserved for symptomatic cases where intervention is being considered and there are either diagnostic or anatomical uncertainties [12].

\subsection{Therapeutic Options}

Management can be split into medical and interventional approaches.

\subsubsection{Medical Management}

Medical management is similar in many ways to stroke caused by atherosclerotic disease however the evidence base for this approach is somewhat lacking. In the first instance antiplatelet therapy is recommended, however the American Stroke Association (ASA) guidelines do not state the optimum drug or dosing regime [18]. There have been no randomised controlled trials in this area. Secondly, antihypertensive treatment is recommended with Angiotensin-converting enzyme inhibitors (ACEi) appearing to be agents of choice [12]. In the context of FMD renal function should be closely monitored following the initiation of an ACEi. Finally there is likely to be benefit in addressing cardiovascular risk factors including lifestyle modification as many patients may have dual pathology and furthermore there may be an association with current cigarette use.

\subsubsection{Interventional Management}

The benefits of revascularisation in cerebrovascular FMD is less clear cut however the recent ASA guidelines suggest that revascularisation is not recommended in asymptomatic carotid artery FMD regardless of the severity of the stenosis [18]. In symptomatic patients with ischaemic symptoms related to the ipsilateral carotid artery may warrant intervention although some may give a trial of antiplatelets therapy and only use percutaneous transluminal angioplasty (PTA) in those with recurrent ischaemic events often in the setting of dissection or in who antiplatelet therapy is contraindicated. Another indication for PTA is for carotid or vertebral pseudoaneurysms when they become symptomatic or are demonstrably increasing in size [18].

\subsection{Follow up}

Annual non-invasive follow up is recommended, however the length between interval scans can be lengthened once the disease is deemed stable [18]. 


\section{Learning Points}

Our case highlights the importance of considering FMD as a potential cause of ischaemic stroke, particularly in women, where traditional vascular and other relevant risk factors have been effectively excluded. Carotid Doppler, often used as a first line investigation, particularly in district general hospitals in England is not an ideal imaging modality to detect FMD. CTA and MRA have much higher sensitivity and specificity and should be considered in selected cases. Once identified, neurosurgical and neuroradiology colleagues should be involved as select cases may benefit from intervention.

\section{References}

[1] Plouin, P.F., Perdu, J., La Batide-Alanore, A., Boutouyrie, P., Gimenez-Roqueplo, A.P. and Jeunemaitre, X. (2007) Fibromuscular Dysplasia. Orphanet Journal of Rare Diseases, 2, 28. http://dx.doi.org/10.1186/1750-1172-2-28

[2] Persu, A., Touzé, E., Mousseaux, E., Barral, X., Joffre, F. and Plouin, P.F. (2012) Diagnosis and Management of Fibromuscular Dysplasia: An Expert Consensus. European Journal of Clinical Investigation, 42, 338-347. http://dx.doi.org/10.1111/j.1365-2362.2011.02577.x

[3] Cragg, A.H., Smith, T.P., Thompson, B.H., Maroney, T.P., Stanson, A.W., Shaw, G.T., Hunter, D.W. and Cochran, S.T. (1989) Incidental Fibromuscular Dysplasia in Potential Renal Donors: Long-Term Clinical Follow-Up. Radiology, 172, 145-147. http://dx.doi.org/10.1148/radiology.172.1.2662248

[4] Hendricks, N.J., Matsumoto, A.H., Angle, J.F., Baheti, A., Sabri, S.S., Park, A.W., et al. (2014) Is Fibromuscular Dysplasia Underdiagnosed? A Comparison of the Prevalence of FMD Seen in CORAL Trial Participants versus a Single Institution Population of Renal Donor Candidates. Vascular Medicine, 19, 363-367. http://dx.doi.org/10.1177/1358863X14544715

[5] Neymark, E., LaBerge, J.M., Hirose, R., Melzer, J.S., Kerlan Jr., R.K., Wilson, M.W. and Gordon, R.L. (2000) Arteriographic Detection of Renovascular Disease in Potential Renal Donors: Incidence and Effect on Donor Surgery. Radiology, 214, 755-760. http://dx.doi.org/10.1148/radiology.214.3.r00mr18755

[6] Olin, J.W., Froehlich, J., Gu, X., Bacharach, J.M., Eagle, K., Gray, B.H., et al. (2012) The United States Registry for Fibromuscular Dysplasia: Results in the First 447 Patients. Circulation, 125, 3182-3190. http://dx.doi.org/10.1161/CIRCULATIONAHA.112.091223

[7] Sang, C.N., Whelton, P.K., Hamper, U.M., Connolly, M., Kadir, S., White, R.I., et al. (1989) Etiologic Factors in Renovascular Fibromuscular Dysplasia: A Case-Control Study. Hypertension, 14, 472-479. http://dx.doi.org/10.1161/01.HYP.14.5.472

[8] Leadbetter, W. and Burkland, L. (1938) Hypertension in Unilateral Renal Disease. Journal of Urology, 39, 611-626.

[9] McCormack, L., Hazard, J. and Poutasse, E. (1958) Obstructive Lesions of the Renal Artery Associated with Remediable Hypertension. American Journal of Pathology, 34, 582.

[10] Harrison Jr., E.G. and McCormack, L.J. (1971) Pathologic Classification of Renal Arterial Disease in Renovascular Hypertension. Mayo Clinic Proceedings, 46, 161-167.

[11] Stanley, J.C., Gewertz, B.L., Bove, E.L., Sottiurai, V. and Fry, W.J. (1975) Arterial Fibrodysplasia: Histopathologic Character and Current Etiologic Concepts. Archives of Surgery, 110, 561-566. http://dx.doi.org/10.1001/archsurg.1975.01360110107018

[12] Olin, J., Gornik, H., Bacharach, J.M., Biller, J., Fine, L.J., Gray, B.H., et al. (2014) AHA Scientific Statement Fibromuscular Dysplasia: State of the Science and Critical Unanswered Questions. A Scientific Statement from the American Heart Association. Circulation, 129, 1048-1078. http://dx.doi.org/10.1161/01.cir.0000442577.96802.8c

[13] Mettinger, K.L. (1982) Fibromuscular Dysplasia and the Brain, II: Current Concept of the Disease. Stroke, 13 , 53-58. http://dx.doi.org/10.1161/01.STR.13.1.53

[14] Cloft, H.J.A., Kallmes, D.F., Kallmes, M.H., Goldstein, J.H., Jensen, M.E. and Dion, J.E. (1998) Prevalence of Cerebral Aneurysms in Patients with Fibromuscular Dysplasia: A Reassessment. Journal of Neurosurgery, 88, 436-440. http://dx.doi.org/10.3171/jns.1998.88.3.0436

[15] Rountas, C., Vlychou, M., Vassiou, K., Liakopoulos, V., Kapsalaki, E., Koukoulis, G., et al. (2007) Imaging Modalities for Renal Artery Stenosis in Suspected Renovascular Hypertension: Prospective Intraindividual Comparison of Color Doppler US, CT Angiography, GD-Enhanced MR Angiography, and Digital Subtraction Angiography. Renal Failure, 29, 295-302. http://dx.doi.org/10.1080/08860220601166305

[16] Anzidei, M., Napoli, A., Zaccagna, F., Di Paolo, P., Saba, L., Cavallo Marincola, B., et al. (2012) Diagnostic Accuracy of Colour Doppler Ultrasonography, CT Angiography and Blood-Pool-Enhanced MR Angiography in Assessing Carotid Stenosis: A Comparative Study with DSA in 170 Patients. La Radiologia Medica, 117, 54-71. 
http://dx.doi.org/10.1007/s11547-011-0651-3

[17] Furie, D.M. and Tien, R.D. (1994) Fibromuscular Dysplasia of Arteries of the Head and Neck: Imaging Findings. American Journal of Roentgenology, 162, 1205-1209. http://dx.doi.org/10.2214/ajr.162.5.8166011

[18] Brott, T.G., et al. (2011) ASA/AACF/AHA/AANN/AANS/ACR/ASNR/CNS/SAIP/SCAI/SIR/SNIS/SVM/SVS Guideline on the Management of Patients with Extracranial Carotid and Vertebral Artery Disease: Executive Summary: A Report of the American College of Cardiology Foundation/American Heart Association Task Force on Practice Guidelines, and the American Stroke Association, American Association of Neuroscience Nurses, American Association of Neurological Surgeons, American College of Radiology, American Society of Neuroradiology, Congress of Neurological Surgeons, Society of Atherosclerosis Imaging and Prevention, Society for Cardiovascular Angiography and Interventions, Society of Interventional Radiology, Society of Neurointerventional Surgery, Society for Vascular Medicine, and Society for Vascular Surgery. Circulation, 124, 489-532. http://dx.doi.org/10.1161/CIR.0b013e31820d8d78 